\title{
Correction to: On the relative strength of radiative feedbacks under climate variability and change
}

\author{
Robert Colman ${ }^{1}$ D $\cdot$ Lawson Hanson ${ }^{1}$
}

Published online: 8 January 2018

๑) Springer-Verlag GmbH Germany, part of Springer Nature 2017

\section{Correction to: Clim Dyn (2017) 49:2115-2129 \\ https://doi.org/10.1007/s00382-016-3441-8}

Two errors were discovered in the calculation of decadal feedbacks under RCP8.5: (i) cloud short wave (SW) and total feedbacks were miscalculated; and (ii) surface albedo and SW water vapour feedbacks were swapped when calculating regressions with climate change feedbacks.

The corrections to these feedbacks means that Fig. 2(b) should be replaced with the following figure ( $C$ and $a$ regressions have changed):

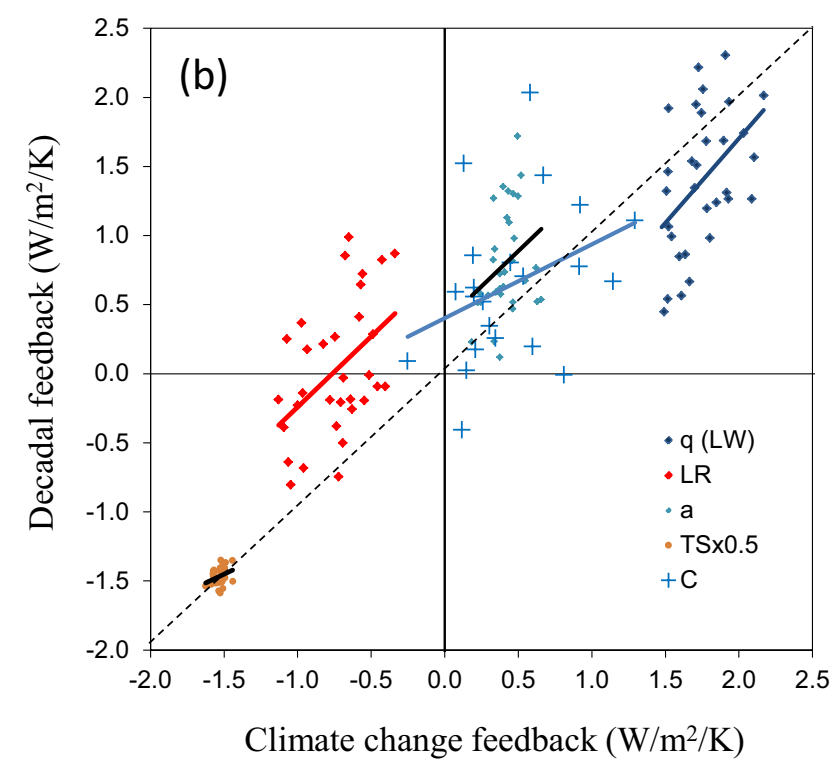

Fig. 2 b Global feedbacks for decadal timescales plotted against corresponding RCP8.5 feedbacks for $\mathrm{LW}$ water vapour $(\mathrm{q}(\mathrm{LW}))$, lapse rate (LR), surface albedo (a), surface temperature (Planck), scaled by a factor of 0.5 (TSx0.5) and total cloud feedback (C). Lines of best fit are shown. Dashed line shows 1:1

The decadal regression coefficients in Table 1 also need correction. The updated center column of this Table is given here (the changed values are for $q(S W), q+L R, a, C$ and $C(S W)$ :
The original article can be found online at https://doi.org/10.1007/ s00382-016-3441-8.

Robert Colman

r.colman@bom.gov.au

1 Australian Bureau of Meteorology, GPO Box 1289,

Melbourne, VIC 3001, Australia 
Table 1 Regression coefficients from linear regression fits of feedbacks from decadal timescales against RCP8.5 feedbacks

\begin{tabular}{lr}
\hline & \multicolumn{1}{c}{ Decadal } \\
\hline$q(L W)$ & $\mathbf{1 . 2 7}(0.47)$ \\
$q(S W)$ & $\mathbf{0 . 9 1}(0.33)$ \\
$L R$ & $\mathbf{1 . 0 7}(0.50)$ \\
$q+L R$ & $0.36(0.15)$ \\
$T S$ & $0.52(0.36)$ \\
$a$ & $\mathbf{1 . 4 0}(0.38)$ \\
$C(S W)$ & $0.02(0.02)$ \\
$C(L W)$ & $-0.03(0.02)$ \\
$C$ & $0.52(0.36)$ \\
\hline
\end{tabular}

Significant coefficients (at the $90 \%$ level) are shown in bold. Correlation coefficients $(r)$ are shown in brackets. Feedbacks shown are: water vapour $(q)$, lapse rate $(L R)$, surface albedo $(a)$, Planck $(T S)$ and cloud feedback $(C)$. For water vapour and clouds, LW/SW components are also shown

In Table 2 the mean strength of the decadal SW and total cloud feedbacks also change. The new values are (in W/ $\left.\mathrm{m}^{2} / \mathrm{K}\right)$ : $\mathrm{C}(\mathrm{SW})$ 0.41, C 0.64 . Standard deviations of intermodel spread in this table are essentially unchanged. LW cloud feedback does not change.

Finally, Figure 8(b) should be replaced with the following figure (in which $\mathrm{C}$ now shows a positive, statistically significant correlation).

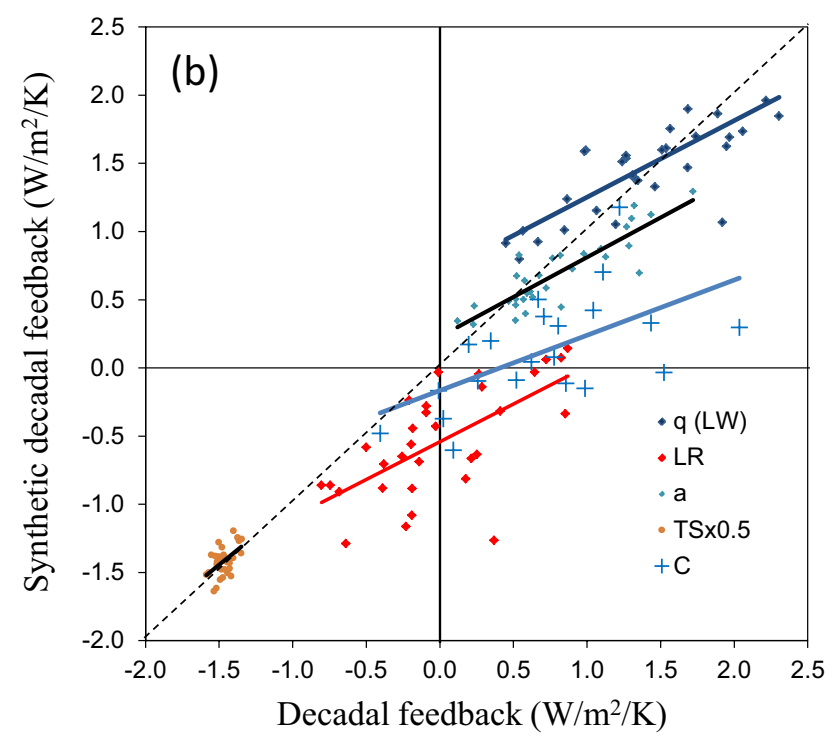

Fig. 8 b Global 'synthetic' feedbacks for decadal timescales plotted against corresponding experiment feedbacks for LW water vapour $(\mathrm{q}(\mathrm{LW}))$, lapse rate (LR), surface albedo (a), surface temperature (Planck), scaled by a factor of 0.5 (TSx0.5) and total cloud feedback (C). Lines of best fit are shown. Dashed line shows 1:1
The corrections also imply small modifications to Fig. 5(b) and (h) and Fig. 7(a) (i.e. in the zonal mean decadal SW and total cloud feebacks). However these changes are minor, and do not change any of the conclusions in the paper, and are therefore not updated here.

Implications of these corrections:

\section{Cloud feedback}

The corrected results in Fig. 2(b) show that there is a positive relationship between total cloud feedback at decadal timescales and that for climate change (RCP8.5). This relationship is statistically significant at the $80 \%$ level (and only narrowly fails significance at the $90 \%$ ). This result therefore bolsters the earlier study of Zhou et al. (2015) that found a strong positive correlation between cloud feedback on interannual timescales and that under climate change. Zhou et al. (2015) identified common physical mechanisms behind the interannual/climate change relationship - and in particular processes due to low cloud cover changes. Such processes increase confidence in the correlation. They should be explored to establish whether they are also operating at decadal timescales, and whether they therefore underlie the positive decadal/climate change cloud feedback correlation found here.

The corrected results in Fig. 8(b) now show a positive correlation between the decadal cloud feedback and the 'synthetic' decadal feedback (calculated by scaling the RCP8.5 cloud feedback by the decadal variability surface temperature pattern). This correlation is statistically significant at the $90 \%$ level and explains $33 \%$ of the variance. This brings clouds 'into line' with the other variables in that scaling of local (climate change) feedback can produce reasonable values of global decadal feedback.

The value of the average total cloud feedback in models under decadal variability, and the SW component, both slightly increase in value (Table 1).

\section{Surface Albedo, SW water vapour feedbacks}

The corrected results in Fig. 2(b) also show that there is a statistically significant relationship between albedo feedback at decadal timescales and that for climate change (RCP8.5). This is now similar to interannual albedo feedback in that both show a significant positive correlation. Short wave decadal water vapour feedback is also significantly correlated with climate change SW water vapour feedback across models (Table 1). 


\section{Conclusions}

The most important change is that corrected values here establish that total cloud feedback at decadal timescales in CMIP5 models shows positive correlations with climate change cloud feedback (consistent with such correlation being previously identified at interannual timescales (Zhou et al. 2015)). The corrections also show there are statistically significant correlations between decadal and climate change feedbacks for surface albedo and SW water vapour. Finally, they show that decadal cloud feedbacks can, to an extent, be reproduced by surface temperature pattern 'scaling' of the climate change feedbacks. Together with the (unchanged) results for interannual feedbacks, these results indicate that both interannual and decadal timescales provide a fruitful direction for future research across a range of feedbacks, including cloud feedback. Where common physical processes are identified, such relationships may potentially provide emergent constraints for climate change feedbacks.

\section{Reference}

Zhou C, Zelinka MD, Dessler AE, Klein SA (2015) Relationship between cloud feedbacks in response to climate change and variability. Geophys Res Lett 42:10463-10469. https://doi.org/10.10 02/2015GL066698 